Para enlazar con este artículo / To link to this article:

http://dx.doi.org/10.6035/MonTI.2019.ne4.9

Para citar este artículo / To cite this article:

Gouttefange, Sébastien. (2019) "Sous-titrer la profusion des « secretions humaines » dans le Playtime de Jacques Tati : une gageure." En: Pérez L. de Heredia, María \& Irene Higes Andino (eds.) 2019. Multilingüismo y representación de las identidades en textos audiovisuales / Multilingualism and representation of identities in audiovisual texts. MonTI Special Issue 4, pp. 253-279

\title{
SOUS-TITRER LA PROFUSION DES « SECRETIONS HUMAINES » DANS LE PLAYTIME DE JACQUES TATI : UNE GAGEURE
}

\author{
Sébastien Gouttefange \\ sebastien.gouttefange@hotmail.fr \\ Université Paul-Valery Montpellier 3
}

\section{Résumé}

Cette étude s'intéresse aux défis multiples que constitue le sous-titrage anglophone de Playtime de Jacques Tati. Comment, s'interroge le traducteur, réconcilier le foisonnement langagier avec l'espace textuel limité, la simultanéité des répliques avec la contrainte d'exposition successive propre au texte écrit, le statut incertain des « sécrétions humaines » du dialogue (entre intelligible et inintelligible) avec un code écrit qui, parce qu'il ne permet pas de jouer sur le volume sonore, impose un choix tranché : traduire ou ne pas traduire? Le chaos dialogique orchestré par Tati constitue un commentaire esthétique sur l'identité sociologique de cette France de l'après-guerre qui entre avec difficulté dans l'ère de la globalisation. Dans les versions professionnelles, les contraintes du sous-titrage mettent-elles à mal la représentation de cette identité ? La créativité des sous-titreurs est-elle au contraire à même de relever le défi posé par le film?

\begin{abstract}
"Subtitling the profusion of the " human secretions » in Jacques Tati's Playtime: a major challenge"

This study aims at throwing light on the challenge that English-speaking translators face when dealing with the subtitling of French filmmaker Jacques Tati's Playtime. How can they possibly reconcile its linguistic abundance with a limited textual space; the overlapping dialogue with the necessity to present information successively when writing a text; the uncertain status of the "human secretions" (alternating between the intelligible and the unintelligible) with the written code, which, as it will not allow a play with volume adjustment, imposes a clear-cut choice: translating or not translating.
\end{abstract}


The dialogic chaos orchestrated by Tati constitutes an aesthetic commentary on the sociological identity of post-war France as the country was painfully entering the era of globalization. In the subtitled versions, do the constraints imposed on the translators hinder a proper representation of this identity or is the subtitlers' creativity able to meet the challenge?

Mots clés : Jacques Tati. Sous-titrage. Playtime. Chevauchement des dialogues.

Keywords: Jacques Tati. Subtitling. Playtime. Overlapping dialogue. 


\section{Introduction}

Un pan entier des recherches récentes en matière de traductologie a abordé la question du sous-titrage essentiellement sous l'angle des contraintes qui pèsent sur cette pratique. Il a, par exemple, clairement été établi que le sous-titrage engendrait une perte de texte de l'ordre de $43 \%$ dans le passage du dialogue parlé vers sa traduction (Pérez-González 1998 : 15). En effet, le texte-cible se doit d'être nettement plus court que le texte parlé dans la mesure où le spectateur doit pouvoir regarder les images tout en lisant les sous-titres, sans toutefois avoir le sentiment d'être confronté à un pur acte de lecture ; cette règle se révèle particulièrement pertinente lorsque le dialogue est singulièrement riche quantitativement parlant (Chiaro 2009 : 148). En lien avec ce premier point, Linde et Kay (1999: 6) évoquent la question des contraintes spatiales (un maximum de deux lignes et aux alentours de 40 caractères pour chaque sous-titre) et temporelles (synchronicité avec la voix parlée, nécessité d'un temps d'exposition suffisant au sous-titre pour permettre au spectateur de lire l'intégralité du texte dans des conditions acceptables). Gottlieb (1994 : 101-3), quant à lui, voit dans le sous-titrage une forme de traduction "diagonale ", dans le sens où il met en relation la langue orale et la langue écrite, contrairement au doublage. Ainsi, avec cette forme de traduction, non seulement il y a adjonction d'un texte à un autre plus que substitution d'un texte à un autre, mais cette co-présence du dialogue original et de sa traduction ajoute une contrainte supplémentaire, tant il est vrai que le spectateur ne manquera sans doute pas de s'étonner si tel dialogue entendu ne fait l'objet d'aucune traduction. La nature volontiers redondante du texte filmique (Georgakopoulou 2009 : 25-9) constitue toutefois un critère objectif justifiant souvent le recours à la réduction quantitative du texte-cible, et donc à la non-traduction. Sur un plan plus qualitatif, Gottlieb (1994 : 105-6) fait encore remarquer que le passage de l'oral à l'écrit engendre inéluctablement des réductions ou modifications qui tiennent à la non-correspondance entre les normes de la langue orale et de la langue écrite (notamment sur les plans grammatical, lexical, syntaxique ou stylistique, sur la question du traitement des pauses ou des faux-départs, des auto-corrections, des répliques prononcées simultanément). Selon Lavaur 
et Şerban (2008: 91), dans le passage du dialogue aux sous-titres, « le plus souvent il y a naturalisation, normalisation et simplification $»$.

À cette première série de servitudes s'en ajoute une deuxième, qui tient à la nature du code employé : les mots écrits imposent une présentation successive de l'information; le sens est construit au fur et à mesure du déroulement de la chaîne écrite, là où le mode d'expression audiovisuel autorise la simultanéité (dialogues qui se chevauchent, conversations simultanées, paroles de chansons mixées avec des dialogues, par exemple), ainsi qu'une appréhension plus synthétique du sens. Diverses stratégies sont employées par les sous-titreurs pour tenter de rendre compte de ces procédés stylistiques cinématographiques : un sous-titre laissant apparaître simultanément une ligne par personnage parlant par exemple, ou des sous-titres cumulatifs (Díaz-Cintas 2005 : 25). Une troisième limite, pour le traducteur, réside dans les attentes du public quant à la nature du dialogue filmique. Il y a fort à parier que ces dernières sont liées au mode de représentation fictionnelle dominant, à savoir le récit classique finalisé, structuré autour d'une logique de causalité, exposant un problème et débouchant sur sa résolution, et propulsé par des ressorts psychologiques (Self 2002 : 45). Dans cette perspective, le dialogue acquiert pour le public (consciemment ou pas) une fonction des plus utilitaires : permettre d'accéder aux pensées des personnages, explorer leurs motivations, faire avancer la narration.

Mais qu'en est-il des films qui osent défier cette logique ? Ceux, qui, depuis la fin des années cinquante, mettent à mal le récit classique, déconstruisent la conception linéaire et causale du temps, acceptent de se trouver aux prises avec la complexité du monde, affirment sans ambages l'impossibilité de mettre à jour les motivations profondes des individus, refusent de réduire le dialogue filmique au rôle qu'il joue au théâtre ou en littérature (Thoret 2006). Playtime (1967), l'œuvre-phare du cinéaste français Jacques Tati, peut constituer un support intéressant pour aborder cette question. En effet, il représente un défi indubitable du point de vue des trois éléments que nous mentionnions ci-dessus. Premièrement, ce qui caractérise ce film sans réel contenu narratif (il est constitué de six séquences organisées autour de différents lieux parisiens) et sans personnage principal digne de ce nom, c'est l'extraordinaire foisonnement de ses dialogues, qui ne peut qu'incommoder un traducteur soumis à des contraintes drastiques en termes d'espace textuel. Deuxièmement, parce que Tati est un artiste qui fuit les gros plans - exception faite de Parade (1974) - et privilégie systématiquement le plan large et la profondeur de champ, c'est aussi le cinéaste de la multitude et de la simultanéité au sein du cadre. Non seulement certains plans de Playtime font penser, de par leur richesse et 
la profusion des détails qu'ils mettent en scène, à certains tableaux de Bruegel l'Ancien (Laufer 2002 : 85), mais leur lecture requiert un dépassement de l'appréhension chronologique ou causale classique : en effet, si plusieurs scènes se déroulent en même temps, aucun principe organisateur ne prend plus en charge la construction du sens pour le spectateur : c'est à lui de procéder aux repérages, aux rapprochements temporels, spatiaux, causaux qu'il juge pertinents. Troisièmement, traduire Playtime est aussi une gageure parce que Tati questionne la fonction traditionnellement attribuée au dialogue dans les films. Celui qui disait : " Moi, j’ai mis le dialogue à l'intérieur du son » (Dondey 2002 : 163) avait en effet une vision toute personnelle des répliques de cinéma, qu'il expose en 1977 dans l'émission de radio Les grandes heures :

J'ai stylisé le dialogue. En stylisant le dialogue, on n'entend que des petites phrases [...]. Quand vous êtes dans un café, vous entendez « deux bières ». On sait pas pour qui sont les bières, on sait pas qui a commandé les bières. Ce n'est pas une construction dramatique. C'est juste une réalité dans l'emploi du dialogue [...]. Il [le public] a besoin d'entendre le dialogue, or moi je le lui ai supprimé. Je lui ai simplement fait un peu comme on appelle en peinture de l'impressionnisme. Je suis parti un peu dans les impressions de dialogue [...].

Comme le rappelle Guerand (2007 : 176), Tati s'inscrit contre l'académisme du cinéma français de l'époque (caractérisé par ses énoncés toujours intelligibles et construits dans le plus pur respect de la grammaire savante, ses répliques véhiculant des idées profondes, l'absence de parasitage par d'autres bruits ou discussions), dont une incarnation possible est la réplique d'anthologie : il s'agissait surtout pour la plupart des artistes de "faire mouche " avec le texte. Chion (2009 : 48) va peut-être encore plus loin que le cinéaste lui-même lorsqu'il affirme qu'il n'y a aucun véritable dialogue dans l'œuvre de Tati, dans le sens où c'est la fonction référentielle de la réplique qui est remise en cause. Au-delà de l'usage théâtral et radiophonique que l'on fait du dialogue cinématographique, le critique argue du fait qu'il existe " pour Tati, pour Fellini, pour Tarkovski [...] un troisième type d'emploi du dialogue, comme sécrétion humaine, parole partiellement intelligible et perdue, qui s'écoule de l'homme " (c'est nous qui soulignons). Le projet de Tati est donc moins d'illustrer la fonction communicative du langage que de mettre en scène l'incommunicabilité qu'il perçoit dans cette France de la fin des années 60 , dans laquelle on voit pourtant déjà poindre la mondialisation des échanges propre à notre époque. Selon Stéphane Goudet (2002 : 25) par exemple, si la parole marque chez Tati les efforts accomplis par les personnages pour établir des échanges, elle est aussi le signe d'une fausse communication, d'où la présence de dialogues qui se chevauchent, de phrases inachevées, inaudibles, 
interminables, ou superfétatoires (les dialogues volontairement redondants par rapport à l'image). Ces « sécrétions humaines » se caractérisent aussi par un étonnant multilinguisme (français, anglais, allemand, italien et espagnol). Notons que Tati choisit, dans la version originale, de ne pas traduire les segments en langue étrangère, pour mieux plonger le spectateur dans le Paris de l'époque : il le place ainsi dans la situation dans laquelle se trouvent eux-mêmes ces personnages limités par leurs connaissances linguistiques, potentiellement « menacés » par des cultures et des langues qui ne font pas encore totalement partie de leur quotidien. C'est en partie ainsi que Tati construit cette identité parisienne un peu caricaturale, faite d'un amalgame de multiples fragments de français et de langues étrangères. Comme l'écrivent Ede et Goudet (2002: 23) : « Tati dialoguiste [...] pousse un peu plus la désarticulation du langage et crée un système linguistique mixte d'une grande drôlerie, où l'on parle le 'sabir atlantique' évoqué par Etiemble dans son livre : Parlez-vous franglais? ?.

Le concept de "profusion " utilisé dans le titre de l'étude nous paraît particulièrement adapté pour qualifier le dialogue tatiesque. Ses différentes acceptions renvoient en effet aux caractéristiques dialogiques que nous avons mises en avant jusqu'à présent. D'abord, le concept fait référence à l'idée de grande abondance. Ensuite, il peut aussi être synonyme d'excès (« une profusion de règlements », par exemple). Enfin, il n'est pas interdit de voir dans son étymologie (le mot latin " profundere », qui signifie " répandre, écouler ») l'idée de débordement des espaces, de porosité des frontières. Si profusion dialogique il y a, comment donc, s'interroge le sous-titreur de Playtime, la préserver lors de la traduction? Comment réconcilier le foisonnement langagier avec l'espace textuel limité, la simultanéité des répliques avec la contrainte d'exposition successive propre au texte écrit, le statut incertain des « sécrétions humaines » (entre l'intelligible et l'inintelligible) avec un code écrit qui, parce qu'il ne permet pas de jouer sur le volume sonore, impose un choix tranché : traduire ou ne pas traduire?

À travers les effets de défocalisation - la dislocation du récit linéaire et finalisé, la presque totale disparition du personnage principal au profit des secondaires, les dialogues envisagés, pour reprendre la formule du cinéaste, comme des "fenêtres ouvertes » sur le monde (Guerand 2007 : 307) — et de refocalisation - la mise en avant d'une réplique par rapport à une autre au moment de l'étape de post-synchronisation) —, la multiplication et la juxtaposition des langues étrangères (qui viennent relativiser l'importance de la langue maternelle), Tati nous incite à penser qu'il vise dans ce film un niveau d'abstraction encore jamais atteint dans les précédents. Il s'agit en effet moins de dérouler un fil narratif structuré autour de différents espaces que de donner 
à observer la ville de Paris, grouillante de vie ; moins de se focaliser sur les actions de quelques personnages que de rendre compte de la société parisienne à la fin de la décennie 60. Les « secrétions humaines » des dialogues de Tati constituent donc un commentaire esthétique sur l'identité sociologique de cette France de l'après-guerre qui entre avec difficulté dans l'ère de la globalisation. Dans les versions professionnelles, les contraintes du sous-titrage mettent-elles à mal la représentation de cette identité ? La créativité des sous-titreurs est-elle au contraire à même de relever le défi posé par le film ?

\section{Cadre théorique et méthodologique}

Nous adoptons un parti pris profondément pragmatique dans cette étude. Nous voulons en effet montrer que non seulement le sens du dialogue de Playtime est indissociable du contexte que constituent les divers systèmes sémiotiques associés au film, mais que le cadre esthétique dans lequel il s'inscrit modifie radicalement la fonction habituellement conférée aux répliques cinématographiques. Dès 1983, Toury montrait que la notion d'équivalence pouvait être complétée par celle de traits pertinents (relevant features). Selon lui, la traduction nous conduisant par nature à des choix, elle débouche nécessairement sur une hiérarchisation des éléments jugés les plus pertinents dans le texte-source (Toury 1983: 117), hiérarchie servant potentiellement de base à la sélection des éléments conservés dans le texte traduit. L'une des caractéristiques essentielles de ce concept est sa relativité : un élément n'est jugé pertinent que par rapport à d'autres traits qui le sont moins. Pour reprendre l'exemple qu'il développe dans son article, si des personnages parcourent un chemin qui a la forme d'une lettre de l'alphabet, le choix du rendu de cette lettre dans une autre langue (et donc le mode de présentation du chemin parcouru) sera plus ou moins important en fonction de la valeur symbolique que prend ou non cette lettre dans le roman et de l'impact éventuel que l'option de traduction peut avoir sur d'autres éléments textuels. Dans la même perspective, nous arguerons du fait que dans une œuvre d'art, le facteur esthétique coiffe tous les autres ; s'agissant de Playtime en particulier, nous défendons l'idée selon laquelle la fonction esthétique du dialogue prime le plus souvent sur sa fonction communicative.

Létude se fixe pour objectif de déterminer si les traductions de Playtime réalisées pour les versions DVD rendent honorablement justice à l'esthétique de ce film. Pour ce faire, elle mettra en perspective deux sous-titrages professionnels du film de Jacques Tati. Le premier est édité par BFI (British Film Institute) et date de 2010. Il n'est fait référence à aucune entreprise de traduction dans ce premier DVD. Le second a été réalisé par Eclair Group en 2014 et il est édité par StudioCanal. Aucun nom de traducteur n'est disponible pour ces deux 
versions. À la lumière des remarques établies précédemment, nous faisons les hypothèses suivantes, que l'étude permettra de confirmer ou d'infirmer :

- Compte tenu des différences de nature entre le dialogue parlé et le sous-titre d'une part, des contraintes d'espace et des différences de traitement de l'information entre un spectateur soumis à un dialogue parlé et un spectateur soumis à des sous-titres d'autre part, de la nature des dialogues de Tati enfin (très prolifiques dans certaines scènes, parfois inaudibles et faisant souvent appel au chevauchement), nous pouvons faire l'hypothèse d'une réduction du dialogue encore plus drastique que celle que l'on rencontre en moyenne dans les films sous-titrés, ainsi que d'une simplification et d'une reformulation plus radicales.

- Compte tenu de l'utilisation massive par Tati des répliques qui se chevauchent, on peut aussi supposer que le sous-titreur, soumis à la contrainte de la présentation successive des informations, sera bien mal armé pour rendre compte de cette simultanéité informationnelle, pourtant particulièrement porteuse de sens. Il est aussi vraisemblable que l'effet produit sur le spectateur relèvera de l'appréhension analytique plutôt que synthétique (des mots alignés les uns à la suite des autres sur l'axe syntagmatique).

- Compte tenu du basculement fréquent chez Tati entre le premier plan et l'arrière-plan sonore, techniquement rendu par l'utilisation d'un volume sonore plus important sur les segments à mettre en valeur, ainsi que de l'absence d'équivalence de cet effet dans le cadre d'un sous-titrage (en dehors de l'utilisation de caractères majuscules, qui reste rare), on peut prédire que le sous-titreur en sera réduit soit à passer sous silence le segment le moins audible, soit à opérer une équivalence entre volume sonore et priorité de l'information (à l'écran, apparaîtront d'abord les informations au volume sonore plus important, puis les autres).

- Enfin, compte tenu de la fonction très particulière prise par le dialogue dans le film, nous pouvons supposer qu'un sous-titrage qui se voudrait très proche de la source en tentant de rendre un maximum de répliques, risquerait de produire un texte qui ferait fi de la dimension de « sécrétion humaine » du dialogue en focalisant l'attention du spectateur sur des éléments dont la fonction n'est pas prioritairement référentielle. Dans cette perspective, l'effet produit sur le spectateur pourrait aller à l'encontre du projet esthétique de Tati. 
Précisons enfin que nous choisissons dans cette étude d'aborder la question du sous-titrage de Playtime de manière résolument descriptive et non normative. Il n'est certes pas question de céder à la tentation de mettre sur le même plan tous les choix de traduction : le projet artistique exigeant de Tati ne saurait s'accommoder d'un quelconque arbitraire. On s'abstiendra toutefois de juger pour s'efforcer de mieux comprendre : il est en effet évident que bien que nous considérions personnellement le facteur esthétique comme central dans le domaine de la traduction artistique, la dégradation des conditions de travail des sous-titreurs ces dernières années (Cornu 2011 : 22-23) constitue un facteur à même de peser fortement sur les choix de traduction et pourrait peut-être expliquer parfois une certaine forme de radicalité dans le rendu. Nous organiserons notre propos autour de quatre stratégies possibles pour le sous-titrage de Playtime : la non-traduction, la reformulation et la simplification, la (dé-/ re-)focalisation, les traductions qui se veulent proches du texte-source.

\section{La non-traduction}

La stratégie de non-traduction occupe une place de choix dans le sous-titrage de Playtime. D'abord, parce que la langue du film se présente souvent comme un flux de signifiants, marqueurs d'une part de cette langue réduite à une « sécrétion humaine ", d'autre part de l'étrangeté qu'une culture peut présenter par rapport à une autre. Le deuxième facteur tient à la nature du sous-titrage : lorsque le nombre de répliques se fait pléthorique, l'image aide parfois grandement à la compréhension et rend du même coup la traduction de certains passages superflue. La troisième cause est à rechercher dans la présence de multiples langues étrangères (à Orly, pendant l'exposition internationale, au restaurant Le Royal Garden) : dans la version originale destinée au public français, Jacques Tati fait le choix de ne pas traduire ces langues, d'où une stratégie similaire chez les sous-titreurs la plupart du temps. Enfin, le dernier facteur est l'inaudibilité volontaire de certaines répliques: elle constitue une contrainte absolue pour le traducteur puisqu'elle se situe au-delà du choix de traduction.

On constate, à l'étude des sous-titrages professionnels, que certaines formes de non-traduction portent sur des segments qui semblent avoir une fonction narrative ou explicative dans la version originale créée par Tati. Ces traductions ont évidemment un impact sur la manière dont le spectateur reconstruit la carte d'identité sociologique du Paris de Tati. Il en va ainsi pour l'exemple 1. Il s'agit d'une annonce au haut-parleur à l'attention du personnage de M. Giffard : 
Exemple 1. BFI $00: 21: 45$; EG $00: 21: 12$

\begin{tabular}{|l|l|l|}
\hline DIALOGUE D'ORIGINE & TRADUCTION DE BFI & $\begin{array}{l}\text { TRADUCTION D'ECLAIR } \\
\text { GROUP }\end{array}$ \\
\hline $\begin{array}{l}\text { - Monsieur Giffard. } \\
\text { - Oh } !\end{array}$ & N.T. $^{1}$ & Mr Giffard. Düsseldorf calling.// \\
\hline
\end{tabular}

On notera, pour commencer, que cet appel a bel et bien une fonction narrative : l'annonce et le départ précipité de Giffard sont causalement associés par Tati puisque Giffard, censé faire passer un entretien d'embauche à Hulot, est appelé au téléphone. Il ne peut par conséquent plus continuer à chercher Hulot comme il le faisait jusqu'alors. La non-traduction de BFI pourrait s'expliquer par l'assimilation de ce segment à l'un des nombreux bruits de fond audibles dans les scènes se déroulant à l'aéroport d'Orly. Toutefois, il faut bien avouer qu'elle peut difficilement permettre aux spectateurs de comprendre le brusque changement de comportement de Giffard. Ils pourraient par exemple le mettre sur le compte de sa surcharge de travail, évoquée dans un précédent énoncé : «Just a moment, I'm very busy » (00:20:43). Si cette attribution causale n'est pas fondamentale, la non-traduction tend à déplacer les raisons pour lesquelles Giffard ne parvient pas à rencontrer Hulot : là où Tati pointe du doigt un monde dans lequel l'individu est aliéné par son travail, qui l'empêche d'accorder la moindre attention aux autres, l'énoncé retenu laisse penser qu'il décide simplement de vaquer à d'autres occupations.

Par ailleurs, si l'esthétique de Tati vise à la déhiérarchisation — de la parole vis-à-vis du bruit (Dondey 2002 : 163), des acteurs professionnels/des vedettes vis-à-vis des amateurs (Guerand $2007: 124)$, du personnage principal vis-à-vis des secondaires (Fieschi 1996 : 16), du premier plan vis-à-vis de l'arrière-plan (Laufer 2002 : 148) — le monde social qu'il dépeint est lui profondément stratifié. Ainsi, Tati de s'amuser de l'invisibilisation des classes sociales modestes, de leur «effacement » dans les lieux fréquentés par le beau monde, comme dans l'exemple 2. Au début de la scène, l'architecte du restaurant The Royal Garden donne quelques informations au patron de l'établissement au sujet de

1. On donnera ici la signification des symboles utilisés dans l'étude. N.T. : non traduit ; « / » signifie que des répliques sont prononcées simultanément ; «// », placé après un segment, indique que celui-ci apparaît normalement sur la même ligne dans le soustitre ; " ? », qu'une partie du dialogue n'est pas audible ; (ALL), que des paroles sont prononcées en allemand ; EG : Eclair Group. Lorsque les cases centrales ou celles de droite sont subdivisées en deux ou plusieurs lignes, on en déduira que la réplique fait l'objet de plusieurs sous-titres. 
la décoration lorsqu'il s'aperçoit, à quelques secondes de l'ouverture de l'établissement, que des ouvriers sont encore en plein travail :

Exemple 2. BFI $01: 02: 24 ;$ EG $01: 01: 52$

\begin{tabular}{|l|l|l|}
\hline DIALOGUE D'ORIGINE & TRADUCTION DE BFI & $\begin{array}{l}\text { TRADUCTION D'ECLAIR } \\
\text { GROUP }\end{array}$ \\
\hline $\begin{array}{l}\text { - Croyez-moi, ça va } \\
\text { avoir beaucoup d'allure, } \\
\text { car je vais vous entourer } \\
\text { l'orchestre de spotlights! }\end{array}$ & $\begin{array}{l}\text { The orchestra will be } \\
\text { spotlighted // }\end{array}$ & It'll look great. \\
\cline { 3 - 3 } - Des clients! & N.T. & $\begin{array}{l}\text { I'll surround the band } \\
\text { with spotlights. }\end{array}$ \\
\hline - Pardon? & & Customers. \\
- Ah ! Oui ! clients! & & \\
\hline
\end{tabular}

BFI propose une non-traduction de la deuxième partie de la conversation. Il n'est toutefois pas certain que cela soit à même de permettre à un non-francophone de comprendre que le patron du restaurant ne souhaite pas laisser voir à ses clients l'envers du décor, à savoir les ouvriers, les outils, les travaux non terminés. Même si les images montrent l'architecte se retournant en direction desdits clients, on est en droit de se demander si le motif de la colère du propriétaire est très clair, au moins au début de la scène. La non-traduction risque donc potentiellement de passer sous silence la dualité que le film construit entre les personnes qui comptent et les «invisibles».

Le microcosme que dépeint Tati se présente aussi comme celui de la communication moderne, alors qu'il est surtout caractérisé par une surenchère verbale qui, en fin de compte, relève de l'incapacité à échanger. Considérons les exemples 3, 4 et 5 . Les énoncés qui suivent constituent dans le film de simples fragments de conversation :

Exemple 3. BFI $00: 05: 15$; EG $00: 04: 43$

\begin{tabular}{|l|l|l|}
\hline DIALOGUE D'ORIGINE & TRADUCTION DE BFI & $\begin{array}{l}\text { TRADUCTION D'ECLAIR } \\
\text { GROUP }\end{array}$ \\
\hline $\begin{array}{l}\text { - (?). } \\
\text { - Ne t'inquiète pas, j'ai } \\
\text { tout mon temps. (?) je } \\
\text { téléphone à Pierre. } \\
\text { - (?). }\end{array}$ & N.T. & And call Pierre. \\
\hline
\end{tabular}


Exemple 4. BFI $00: 06: 54$; EG $00: 06: 19$

\begin{tabular}{|c|c|c|}
\hline DIALOGUE D'ORIGINE & TRADUCTION DE BFI & $\begin{array}{l}\text { TRADUCTION D'ECLAIR } \\
\text { GROUP }\end{array}$ \\
\hline \multirow{4}{*}{$\begin{array}{l}\text { - Non, non. } \\
\text { - Quelques mots pour } \\
\text { notre journal! } \\
\text { - Non, non. / - Juste } \\
\text { un mot, juste un mot, } \\
\text { juste un mot, s'il vous } \\
\text { plaît. } \\
\text { - Monsieur le } \\
\text { Président? } \\
\text { - Non, non, merci. (?) } \\
\text { tout ce que j'ai à dire } \\
\text { pour l'instant (?). }\end{array}$} & \multirow[t]{4}{*}{ N.T. } & A few words for my paper. \\
\hline & & Just a word, please! \\
\hline & & Mr President! \\
\hline & & All I can say now is... \\
\hline
\end{tabular}

Exemple 5. BFI $00: 45: 29$; EG $00: 44: 56$

\begin{tabular}{|l|l|l|}
\hline DIALOGUE D'ORIGINE & TRADUCTION DE BFI & $\begin{array}{l}\text { TRADUCTION D'ECLAIR } \\
\text { GROUP }\end{array}$ \\
\hline $\begin{array}{l}\text { Pour vos moquettes, } \\
\text { pour vos tapis, pour } \\
\text { vos carpettes, un seul } \\
\text { produit (?). }\end{array}$ & N.T. & For your rugs and carpets, \\
\cline { 2 - 3 } & & a single product... \\
\hline
\end{tabular}

Dans le sous-titrage de BFI, on constate que ces énoncés ne sont pas rendus en anglais (ces trois exemples sont tout à fait représentatifs du recours fréquent à la non-traduction tout au long du film chez ce sous-titreur). Le traducteur semble les assimiler aux "sécrétions humaines » dont parle Chion : il s'agit alors d'évacuer tous les éléments jugés encombrants parce que non narratifs. La réplique : " tout ce que j'ai à dire pour l'instant » ne constitue qu'une partie de phrase puisque le début et la fin de l'énoncé ne sont pas audibles. Dans le premier énoncé en gras, il s'agit d'une passagère qui ne fait que traverser l'écran et qu'on ne reverra plus par la suite; dans le troisième, d'une publicité, vraisemblablement entendue dans un bus ou sur l'autoradio d'une voiture. Il est intéressant toutefois de constater que le traducteur d'EG se positionne différemment. On ne peut évidemment pas avoir de position étroitement technicienne pour évaluer la pertinence de ces options, qui ont chacune leurs avantages et leurs inconvénients : la restitution permet sans doute de donner à entendre des fragments de conversation normalement accessibles au public français : en ce sens, elle participe à la construction de l'identité de l'époque ; en contrepartie, elle risque de surcharger le sous-titrage et de rendre plus 
délicate la lecture attentive de l'image, si importante dans ce film. Mais ce Paris de la fin de la décennie 60 n'est pas seulement celui de la surcharge verbale : c'est aussi tout simplement celui de la non-communication, comme l'indique l'exemple 6. Dans la situation suivante, un homme venant de la rue s'approche du vieux gardien à Orly, qui se trouve, lui, à l'intérieur du bâtiment, pour lui demander du feu :

Exemple 6. BFI $00: 12: 30$; EG $00: 11: 56$

\begin{tabular}{|l|l|l|}
\hline DIALOGUE D'ORIGINE & TRADUCTION DE BFI & $\begin{array}{l}\text { TRADUCTION D'ECLAIR } \\
\text { GROUP }\end{array}$ \\
\hline - (?). & N.T. & Thanks, chief! \\
\cline { 2 - 2 } $\begin{array}{l}\text { mais va par là... C'est ça, } \\
\text { mmmm. }\end{array}$ & & So long, guys! \\
\hline $\begin{array}{l}\text { - Merci bien, chef ! Au } \\
\text { revoir, Messieurs! }\end{array}$ & & \\
\hline
\end{tabular}

Si l'on peut admettre que le dialogue présente bien un faible caractère narratif ou explicatif, la non-traduction de BFI est tout de même au moins problématique dans le sens où elle fait fi du discours sur l'incommunicabilité. En effet, si le début de la scène est appréhendé du point de vue du gardien (il est sur les plans visuel et auditif la source perceptive), à la fin, c'est l'homme à l'extérieur qui devient le focalisateur. Tati choisit de pointer les difficultés de communication en mettant en scène des personnages enfermés dans leur point de vue respectif. La première traduction a pour effet de reléguer ce dialogue au rang de bruit de fond et il est bien difficile, du coup, de distinguer ce passage de tous ceux qui font aussi l'objet d'une non-traduction. La seconde, en ne rendant compte que de la parole du deuxième homme, ne permet pas non plus de suggérer ce cloisonnement des points de vue. Il aurait peut-être été judicieux, au début de la scène, de traduire seulement les propos du gardien, et à la fin, de se contenter de ceux de l'homme à l'extérieur.

Un autre phénomène intéressant en lien avec la non-traduction est le traitement des chansons dans le film. Comme toute forme artistique, la chanson au cinéma ne peut avoir de fonction a priori. Ainsi que l'écrit René Prédal (2007: 20-21) à propos du long travelling final des Quatre cents coups (François Truffaut 1959):

Le mouvement d'appareil ne signifie pas en lui-même ; il est indissociable du décor, du personnage et de sa place dans le récit. Il n'existe donc pas de grammaire cinématographique car si le cinéma est un langage, il n'est pas une 
langue et l'on ne peut pas dire que la plongée écrase et que la contre-plongée grandit le personnage.

Dans cette perspective, la fonction de la chanson ne peut être déterminée qu'à partir du contexte dans lequel elle s'insère : telle chanson peut, par exemple, apparaître dans deux films différents et prendre des fonctions radicalement dissemblables (usage intra- ou extra-diégétique, focalisation sur le caractère mélodique de la chanson, sur le texte, sur la capacité qu'elle a de nous replonger dans une époque donnée, etc.). C'est la chanson d'Aristide Bruant, À la Bastille (1889), entonnée par les clients du Royal Garden vers la fin du film, qui fera ici l'objet de notre commentaire. BFI comme EG choisissent tous deux de passer sous silence le texte de la chanson. Du point de vue de leur analyse traductologique du passage, on peut en déduire qu'elle a, dans leur esprit, essentiellement pour fonction de créer une ambiance typiquement française. Cette option est, certes, parfaitement légitime - le défilement intégral du texte de la chanson aurait largement alourdi le sous-titrage—, mais il a le défaut de laisser de côté un point essentiel : la référence à la Bastille a des airs de révolution qui prennent tout leur sens dans le contexte du film. Pour Tati, dans ce Paris des années 60, il s'agit de s'engager dans une "révolution » à la fois sociale et technique : se débarrasser des hiérarchies sociales mortifères et de notre dépendance vis-à-vis du monde des objets. Le cinéaste rappelle également que l'identité française est aussi faite des combats passés, que l'art en porte la trace et que notre mémoire peut inspirer les luttes présentes. Une alternative, non retenue par les traducteurs, aurait été envisageable : se contenter de la traduction du refrain, solution à mi-chemin entre la pesanteur qu'aurait engendrée la traduction intégrale et l'entropie de la non-traduction pure et simple.

Si les exemples présentés jusqu'ici pourraient induire chez le spectateur une construction différente de l'identité sociale parisienne, les traductions retenues par le sous-titreur de BFI - plus radicales dans la mesure où il opte pour la non-traduction d'une majorité des dialogues du film - s'avèrent à bien des égards tout à fait pertinents dans nombre d'occurrences. C'est le cas notamment lorsque le recours à la non-traduction s'explique par la possibilité pour le spectateur de déduire facilement le sens des répliques à partir de la situation. Faute de place, nous n'analyserons ici que le seul exemple 7. Tati met en scène M. Hulot adressant la parole à un passant alors qu'il sort d'un bus : 
Exemple 7. BFI $00: 12: 00 ;$ EG $00: 11: 28$

\begin{tabular}{|l|l|l|}
\hline DIALOGUE D'ORIGINE & TRADUCTION DE BFI & $\begin{array}{l}\text { TRADUCTION D'ECLAIR } \\
\text { GROUP }\end{array}$ \\
\hline $\begin{array}{l}\text { - Pardon, monsieur, s'il } \\
\text { vous plaît ! (?). }\end{array}$ & N.T. & Wait! \\
\cline { 3 - 3 } & & Over there. \\
\hline
\end{tabular}

Le spectateur entend assez distinctement le début de la scène, mais pas la fin de la conversation en raison des bruits du moteur et de la ville. Même pour un non-francophone, les mimiques de Hulot sont suffisamment explicites pour ne laisser aucun doute sur l'objet de sa quête (un renseignement sur sa destination, vraisemblablement), ce qui explique le recours à la non-traduction chez BFI. Mais peut-être faut-il s'interroger plus encore sur le sens de cette scène ? La stratégie de Tati vise-t-elle seulement à alléger son dialogue en ne l'encombrant pas d'éléments superflus, ou doit-on y voir un commentaire sur la ville et ses conditions de vie? Ainsi, si l'on admet que la conversation est sans doute volontairement étouffée par les bruits de la ville, traduire (comme Eclair Group ici) un passage difficilement audible ne va-t-il pas à l'encontre des intentions du cinéaste ? Au final, l'essentiel de la scène réside-t-il dans le contenu de la conversation, ou plutôt dans l'idée que la forme la plus anodine de communication est devenue difficile dans cette ville ultramoderne?

\section{Simplifications et reformulations}

Dans leurs tentatives de traduction de la foisonnante bande-son de Playtime, nous avons constaté que les sous-titreurs avaient régulièrement recours à un rendu qui s'apparentait plus à de la réécriture qu'à de la traduction littérale. On s'intéressera d'abord, avec l'exemple 8, aux changements qui affectent la dimension sociolectale du discours. Dans cette scène, le vieux gardien accueille Hulot et s'efforce de communiquer avec un supérieur par le biais d'une machine qu'on devine très sophistiquée : 
Exemple 8. BFI $00: 12: 52$; EG $00: 12: 19$

\begin{tabular}{|c|c|c|}
\hline DIALOGUE D'ORIGINE & TRADUCTION DE BFI & $\begin{array}{l}\text { TRADUCTION D'ECLAIR } \\
\text { GROUP }\end{array}$ \\
\hline \multirow{5}{*}{$\begin{array}{l}\text { - Ouais, ouais, ouais, } \\
\text { ouais. Ouais, bon, alors } \\
\text { (il s'éclaircit la gorge). } \\
\text { Là, c'est parfait. Alors, } \\
\text { monsieur, si vous voulez } \\
\text { bien, je m'occupe de } \\
\text { vous. J'en ai pour deux } \\
\text { minutes. Voilà. }\end{array}$} & \multirow{5}{*}{$\begin{array}{l}\text { Sit here, I won't be a } \\
\text { moment }\end{array}$} & Yeah... \\
\hline & & O.K. So... \\
\hline & & There. \\
\hline & & If you don't mind, sir... \\
\hline & & This'll only take a minute. \\
\hline \multirow{7}{*}{$\begin{array}{l}\text { Alors, euh... Voyons voir, } \\
\text { euh... (?) bien. Ça... Bon. } \\
\text { (?) voilà. J'appelle. Euh... } \\
\text { Voyons, ah ! Deuxième. } \\
\text { Oui, oui, oui, oui, oui, } \\
\text { oh, oui ! (?) alors. } \\
\text { - (?) Qu'est-ce que } \\
\text { c'est? } \\
\text { - Ah ! (?) Alors, euh... } \\
\text { je demande le } 440 \ldots . . .2 .\end{array}$} & \multirow{7}{*}{$\begin{array}{l}\text { I want four hundred and } \\
\text { forty-...// }\end{array}$} & Let's see... \\
\hline & & I want... \\
\hline & & This one... \\
\hline & & Who is it? \\
\hline & & Well... \\
\hline & & I want $4 \ldots 4 \ldots$ \\
\hline & & $\ldots 2$ \\
\hline Je répète... & I repeat & I repeat... \\
\hline $\begin{array}{l}\text { (Voix basse) } 440 . \text { (Haute } \\
\text { voix) ...2! }\end{array}$ & ...two & $-4 \ldots 4 \ldots$ \\
\hline \multirow{4}{*}{$\begin{array}{l}\text { (La voix particulièrement } \\
\text { peu audible annonce } \\
\text { un chiffre qui pourrait } \\
\text { être aussi bien compris } \\
\text { comme un « un » que } \\
\text { comme un « deux »). } \\
\text { - Non, pas « un », } \\
\text { deux! } \\
\text { (Même son inaudible). } \\
\text { - Ouais ! Alors là, c'est } \\
\text { bien, ça, ça va, ça va, } \\
\text { alors là, ouais, là ! Bon. } \\
\text { Là ! Et voilà ! }\end{array}$} & \multirow[t]{4}{*}{ Not one, two! } & $\begin{array}{l}\text {-2. } \\
\text { - Too? }\end{array}$ \\
\hline & & - Not too! 2! \\
\hline & & $\begin{array}{l}-2 ? \\
- \text { Yes. } \\
\end{array}$ \\
\hline & & There. \\
\hline \multirow{2}{*}{$\begin{array}{l}\text { Tous ces trucs } \\
\text { électriques... À } \\
\text { savoir qu'est-ce qu'y } \\
\text { a là-dedans, faut s'y } \\
\text { connaître. Avec tous leurs } \\
\text { boutons. Eh! (Il siffle). }\end{array}$} & \multirow{2}{*}{$\begin{array}{l}\text { These electrical gadgets } \\
\text { are all very// } \\
\text { well if you know your } \\
\text { way around // }\end{array}$} & $\begin{array}{l}\text { Who understands } \\
\text { all this electronic stuff? }\end{array}$ \\
\hline & & All these buttons. \\
\hline
\end{tabular}

On notera d'abord que c'est l'aspect dénotatif du dialogue (s'asseoir, patienter) qui concentre l'attention de BFI, alors que Tati, via le lexique et le niveau de langue utilisés, s'attache à marquer le contraste chez le gardien entre la 
langue apprise, professionnelle ( «si vous voulez bien, je m'occupe de vous ») et la langue « naturelle», spontanée (" Ouais, ouais, ouais, ouais. Ouais, bon alors »). La caractérisation linguistique du personnage (syntaxe « hachée » et niveau de langue familier d'un côté, syntaxe et lexique neutres de l'autre) est donc altérée, en dépit du fait que le raclement de gorge et la cigarette à la bouche puissent probablement être considérés comme des marqueurs de statut social. En outre, la minutie avec laquelle le cinéaste décrit la lutte du gardien avec la machine et la complexité de l'opération sont sans conteste en grande partie perdues à la traduction. Tati s'attaque ici aux circonvolutions inutiles que la technique nous fait emprunter (alors qu'il ne s'agit finalement que de faire appeler quelqu'un). Dans le dialogue initial, l'effet est créé par la description méticuleuse de chacune des étapes, commentées par le personnage. La traduction se contente du rendu des phases essentielles — même si le jeu du comédien semble compenser partiellement la simplification du dialogue là où les répliques d'origine s'intéressent au détail. La traduction d'EG, elle, rend davantage justice à la dimension sociolinguistique du passage, puisqu'elle travaille plus précisément les questions lexicale (registre familier : "Yeah", «O.K. », "stuff»; marqueurs d'oralité : « There ») et syntaxique (énoncés inachevés, phrases sans verbe : «All these buttons »).

Limportance de la dimension sociolectale transparaît aussi dans l'utilisation que fait Tati des anglicismes, signe d'un certain snobisme (comme chez Proust) comme d'une forme de complexe d'infériorité de cette France vis-à-vis de son cousin américain. Analysons à ce propos l'exemple 9. Le personnage de Schneider vient de retrouver Hulot, son ancien camarade de régiment :

Exemple 9. BFI à partir de $00: 46: 34$; EG à partir de $00: 46: 00$

\begin{tabular}{|c|c|c|}
\hline DIALOGUE D'ORIGINE & TRADUCTION DE BFI & $\begin{array}{l}\text { TRADUCTION D'ECLAIR } \\
\text { GROUP }\end{array}$ \\
\hline \multirow{2}{*}{$\begin{array}{l}\text { - Ah, tu sais, ce trafic, } \\
\text { dis-donc, ouh! Et, } \\
\text { comme ils disent, hein : } \\
\text { « Time is money!». }\end{array}$} & \multirow{2}{*}{$\begin{array}{l}\text { Such traffic! As they say: } \\
\text { Time is money }\end{array}$} & What traffic! \\
\hline & & $\begin{array}{l}\text { But like they say... } \\
\text { Time is money. }\end{array}$ \\
\hline $\begin{array}{l}\text { Alors, tu vois, c'est le } \\
\text { modèle Deluxe. }\end{array}$ & It's the de luxe model & It's the deluxe model. \\
\hline \multirow{2}{*}{$\begin{array}{l}\text { Je l'ai depuis deux jours. } \\
\text { Je l'ai payée cash, hein, } \\
\text { mon vieux! }\end{array}$} & \multirow{2}{*}{$\begin{array}{l}\text { I've had it for two days. } \\
\text { I paid cash down }\end{array}$} & I got it 2 days ago. \\
\hline & & I paid cash. \\
\hline \multicolumn{3}{|l|}{$[\ldots]$} \\
\hline $\begin{array}{l}\text { - Allez, viens mon vieux } \\
\text { ! Je vais te faire visiter } \\
\text { mon home. }\end{array}$ & I'll show you my home & Come see my home. \\
\hline
\end{tabular}




\begin{tabular}{|l|l|l|}
\hline$[\ldots]$ & & \\
\hline - Allez, allez, viens mon & Come in for a moment, & Come in for a drink. \\
\cline { 2 - 3 } vieux, tu vas boire un & have a drop of Scotch & Have a scotch. \\
glass, là. & & \\
- Ah non non. & & \\
- Mais si, quoi. Tu vas & & \\
bien prendre un petit & & \\
scotch là ! Mais si, mais & & \\
si ! Depuis le temps & & \\
qu'on s'est pas vus, là ! & & \\
(?). & & \\
\hline
\end{tabular}

On note ici que le discours de Schneider recèle son lot d'anglicismes, destinés, dans son esprit, à asseoir son statut social : comme dans Jour de fête (1949), le peuple américain est paré de toutes les vertus - notamment sur le plan technique - par certains personnages. Les propositions de BFI et d'Eclair Group ne permettent pas au spectateur d'avoir accès, par le biais de ces marqueurs essentiels, à l'ironie voulue par Tati puisque les traducteurs se contentent d'utiliser les mêmes mots. Mais l'anglais n'est pas la seule langue à laquelle Tati confronte le spectateur sans lui proposer de traduction. On pourrait même affirmer que le Paris de la fin des années 60 est présenté comme particulièrement multilingue. On étudiera, avec l'exemple 10, le cas de l'allemand (mais on gardera à l'esprit que d'autres langues émaillent la bande-son de Playtime). Ici, un employé et son patron (tous deux allemands) échangent au sujet d'un homme qui s'est montré particulièrement sans gêne quelques instants auparavant :

Exemple 10. BFI $00: 35: 06$; EG $00: 34: 32$

\begin{tabular}{|c|c|c|}
\hline DIALOGUE D'ORIGINE & TRADUCTION DE BFI & $\begin{array}{l}\text { TRADUCTION D'ECLAIR } \\
\text { GROUP }\end{array}$ \\
\hline \multirow{4}{*}{$\begin{array}{l}\text { - Guten morgen, Herr } \\
\text { Direktor. (ALL) il avait } \\
\text { un long pipe, il avait un } \\
\text { long écharpe. Il était assis } \\
\text { à le bureau. } \\
\text { - (ALL). } \\
\text { - (ALL) il a fouillé dans } \\
\text { tous les papiers, comme } \\
\text { ça, vous voyez. } \\
\text { - (ALL). } \\
\text { - (ALL) sans se gêner. } \\
\text { - (ALL). } \\
\text { - Ah vraiment ! Je... }\end{array}$} & $\begin{array}{l}\text { When I was busy a man } \\
\text { rifled } \\
\text { the desk drawer }\end{array}$ & N.T. \\
\hline & $\begin{array}{l}\text { He had a long pipe and a } \\
\text { long scarf// }\end{array}$ & $\begin{array}{l}\text { He had a pipe and a long scarf.// } \\
\text { He sat down there. }\end{array}$ \\
\hline & $\begin{array}{l}\text { He went through the } \\
\text { papers }\end{array}$ & $\begin{array}{l}\text { He went through all the } \\
\text { papers.// }\end{array}$ \\
\hline & $\begin{array}{l}\text { He walked off with } \\
\text { some, } \\
\text { cool as you please }\end{array}$ & N.T. \\
\hline
\end{tabular}


Dans cette scène, non seulement les phrases en allemand ne font l'objet d'aucune traduction en anglais — ce que l'on peut juger parfaitement recevable si l'on admet que le spectateur anglophone doit être placé dans les mêmes conditions que son homologue français - mais dans la version BFI, le dialogue anglais semble tout simplement constituer un résumé de ce qui se dit en allemand. Par exemple, les segments "When I was busy » ou « He walked off with some » n'ont pas leur équivalent dans le dialogue français. Il est aussi envisageable que ces deux énoncés résument ce que l'on voit à l'écran. La traduction retenue peut néanmoins légitimement être interrogée puisqu'elle donne une tout autre image du rapport que les langues entretiennent entre elles dans l'univers de Playtime : là où Tati met en scène une juxtaposition des langues qui rend difficile l'appréhension du sens de la scène, le recours au seul anglais dans les deux versions donne, lui, au contraire, un sentiment d'unité et de cohérence linguistique et culturelle. Sur la question de la fidélité au texte de départ, le traducteur d'EG adopte en tout cas une position différente de celle de son concurrent puisqu'on constate qu'il ne s'autorise pas à aller au-delà de ce qui est dit en français.

Dans Playtime, quatre lieux différents évoquent la technicisation progressive du mode de vie: Orly et ses immeubles de verre et d'acier, l'exposition des inventions (la porte que l'on claque silencieusement, le balai lumineux, etc.), l'immeuble moderne de l'ami de régiment de Hulot et le Royal Garden (son décor sophistiqué, sa climatisation). Il n'est donc pas étonnant que le langage des personnages en porte la trace : c'est parfois un véritable technolecte qui transparaît dans le discours des personnages, comme le montre l'exemple 11. Dans la scène ci-dessous, un vendeur présente à un client un modèle de porte censé être révolutionnaire :

Exemple 11. BFI à partir de $00: 33: 57$; EG à partir de $00: 33: 24$

\begin{tabular}{|l|l|l|}
\hline DIALOGUE D'ORIGINE & TRADUCTION DE BFI & $\begin{array}{l}\text { TRADUCTION D'ECLAIR } \\
\text { GROUP }\end{array}$ \\
\hline $\begin{array}{l}\text { - Après vous, monsieur. } \\
\text { — Alors, d'après } \\
\text { vous, vos portes sont } \\
\text { totalement insonores? }\end{array}$ & N.T. & Your doors are soundproof? \\
$\begin{array}{l}\text { - Absolument, } \\
\text { j'explique à vous. }\end{array}$ & & Absolutely. Let me explain. \\
\hline
\end{tabular}




\begin{tabular}{|c|c|c|}
\hline \multirow{4}{*}{$\begin{array}{l}\text { - Regardez. La porte, } \\
\text { elle a fabriquée dans } \\
\text { un matière totalement } \\
\text { insonorisée (?). /_- (?) } \\
\text { Ah, oui, on n'entend } \\
\text { rien lorsqu'elles sont } \\
\text { fermées. Très bien. Très } \\
\text { intéressant. } \\
\text { — Vous entendriez même } \\
\text { pas... } \\
\text { - Mais dites-moi, est-ce } \\
\text { que je peux fermer cette } \\
\text { porte sans qu'elle fasse le } \\
\text { moindre bruit. } \\
\text { - Je vous fais un } \\
\text { démonstration. Grâce à } \\
\text { l'épaisseur de la porte en } \\
\text { Sonex, voyez-vous... }\end{array}$} & \multirow{4}{*}{$\begin{array}{l}\text { This door is made of } \\
\text { sound-proofing // } \\
\text { material }\end{array}$} & $\begin{array}{l}\text { It's made of totally } \\
\text { soundproof materials. }\end{array}$ \\
\hline & & $\begin{array}{l}\text { - Very interesting. } \\
\text { — You can't even hear... }\end{array}$ \\
\hline & & $\begin{array}{l}\text { I can close it } \\
\text { in complete silence? }\end{array}$ \\
\hline & & $\begin{array}{l}\text { Let me show you. } \\
\text { Thanks to this layer of } \\
\text { Sonex...// }\end{array}$ \\
\hline \multicolumn{3}{|l|}{$[\ldots]$} \\
\hline \multirow{3}{*}{$\begin{array}{l}\text { - Auriez-vous } \\
\text { l'obligeance de me } \\
\text { montrer vos prix. } \\
\text { - Ah, ah, alors voici nos } \\
\text { prix et ici les références } \\
\text { de nos différents } \\
\text { articles. } \\
\text { - Je vous remercie. } \\
\text { - A votre disposition, } \\
\text { monsieur. } \\
\text { - Merci. }\end{array}$} & \multirow[t]{3}{*}{ N.T. } & Here are our prices... \\
\hline & & $\begin{array}{l}\text { with the various reference } \\
\text { numbers.// }\end{array}$ \\
\hline & & At your service, sir. \\
\hline
\end{tabular}

On remarquera une nouvelle fois un écart de traitement entre les deux traductions professionnelles. Outre la différence de rendu du détail dans le dialogue, on note bien ici que la traduction d'EG s'efforce de conserver davantage de mots techniques que celle de BFI : soundproof, layer of Sonex, reference numbers, par exemple. Dans la traduction proposée par BFI, c'est donc toute la construction identitaire autour de l'objet technique (le vendeur compétent est celui qui maîtrise le technolecte susceptible de convaincre son auditoire) qui est affaiblie par le biais des non-traductions et des simplifications opérées.

\section{Focalisation, défocalisation, refocalisation}

Comme mentionné plus haut, ce qui caractérise notamment la bande sonore des films de Tati, c'est le bouleversement des hiérarchies qu'il introduit entre les différents éléments. On sait que Tati travaillait systématiquement le son en post-production. Laufer $(2002: 57)$ rapporte par exemple que le cinéaste 
enregistre 365 bruits de vague différents pour la préparation des Vacances de Monsieur Hulot (1953). Il n'y a donc pas de son « réaliste » chez Tati : la bande-son est souvent débarrassée de ses scories et certains éléments sont au contraire mis en valeur. Ce n'est par exemple jamais le positionnement de la caméra par rapport à l'objet ou au personnage qui détermine le volume sonore : seule la composition esthétique décide de ces paramètres. Le traducteur doit donc s'intéresser de près à ces effets de (dé-)focalisation s'il veut rendre compte de l'esthétique tatiesque. Abordons un premier problème avec l'exemple 12. Dans cette conversation entre des personnages qui se rencontrent par hasard au restaurant, chaque convive semble se disputer la parole :

Exemple 12. BFI $01: 14: 50 ;$ EG $01: 14: 17$

\begin{tabular}{|l|l|l|}
\hline DIALOGUE D'ORIGINE & TRADUCTION DE BFI & $\begin{array}{l}\text { TRADUCTION D'ECLAIR } \\
\text { GROUP }\end{array}$ \\
\hline $\begin{array}{l}\text { C Oh / - Oui ! / - } \\
\text { C'est pas vrai / - C'est } \\
\text { pas possible. } \\
\text { — Qu'est-ce que vous } \\
\text { faites ici ? } \\
\text { - Bonjour (?) Henri ! }\end{array}$ & $\begin{array}{l}\text { What are you doing } \\
\text { there? }\end{array}$ & What're you doing here? \\
\hline $\begin{array}{l}\text { - Ce que je suis } \\
\text { contente. J'avais } \\
\text { commandé une } \\
\text { excellente table. Venez } \\
\text { avec nous! (?). }\end{array}$ & $\begin{array}{l}\text { Join us at our excellent } \\
\text { table }\end{array}$ & $\begin{array}{l}\text { We reserved a good table. Come } \\
\text { with us. }\end{array}$ \\
\hline
\end{tabular}

Tati, dans ses films, affiche clairement sa volonté de mixer au même volume sonore plusieurs conversations en même temps, afin à la fois de dynamiser la scène et de traduire l'idée que les échanges ne débouchent sur aucune communication authentique. Les sous-titreurs, eux, contraints par l'écrit, ne peuvent au mieux que juxtaposer les répliques et réduire drastiquement le dialogue pour éviter à la fois l'excès de texte et le manque de clarté. L'effet de simultanéité des conversations fait place à une simple juxtaposition d'énoncés (par exemple, "What are you doing there? / " Join us at our excellent table »).

Dans ce jeu sur la (dé-)focalisation, les décodages partiels ou erronés semblent inévitables. C'est alors le subtil discours sociologique de Tati sur son époque qui risque d'être affaibli ou dénaturé. Etudions à ce propos l'exemple 13. Au restaurant The Royal Garden, le gérant fait de discrètes remontrances 
à son serveur : les clients sont tout autour de lui et il ne faut en aucun cas les déranger :

Exemple 13. BFI $01: 13: 23 ;$ EG $01: 12: 49$

\begin{tabular}{|l|l|l|}
\hline DIALOGUE D'ORIGINE & TRADUCTION DE BFI & $\begin{array}{l}\text { TRADUCTION D'ECLAIR } \\
\text { GROUP }\end{array}$ \\
\hline $\begin{array}{l}\text { (Bruit de la salle de } \\
\text { restaurant. Dialogue très } \\
\text { peu audible). }\end{array}$ & $\begin{array}{l}\text { Waiter, have you } \\
\text { attended } \\
\text { - Garçon, eh bien, (?). } \\
\text { - Ah, non non non non. } \\
\text { Là ! Pas là ! }\end{array}$ & $\begin{array}{l}\text { Waiter. } \\
\text { Can't you handle this table? }\end{array}$ \\
\hline
\end{tabular}

Du point de vue de Tati, il s'agit d'illustrer la question des jeux hiérarchiques qui s'établissent au sein du restaurant (entre le gérant et le serveur, d'une part ; entre les clients et le gérant, d'autre part). Les sous-titreurs, cependant, restituent ces paroles de manière totalement intelligible, négligeant en cela de rendre compte de la dimension sociale de la scène (et de l'humour qui en découle). Il ne s'agit pas en effet de donner à tout comprendre, mais de placer le public dans les conditions voulues par Tati : dans ce contexte précis, le spectateur est assis au milieu du restaurant avec les autres clients et il ne peut exiger de recevoir de signes plus explicites que ceux auxquels les convives ont accès.

Il est bien sûr, en revanche, nombre d'occurrences pour lesquelles il y a coincidence entre les moyens filmiques mis en ouvre pour focaliser l'attention du spectateur et l'emploi d'un sous-titre qui aura la même fonction, comme dans l'exemple 14, renvoyant à une annonce publicitaire radiophonique :

Exemple 14. BFI $00: 55: 57$; EG $00: 55: 23$

\begin{tabular}{|l|l|l|}
\hline DIALOGUE D'ORIGINE & TRADUCTION DE BFI & $\begin{array}{l}\text { TRADUCTION D'ECLAIR } \\
\text { GROUP }\end{array}$ \\
\hline $\begin{array}{l}\text { Mesdames, utilisez Quick } \\
\text { Cleaner, parce que Quick } \\
\text { Cleaner est le... }\end{array}$ & $\begin{array}{l}\text { Ladies, use Quick Cleaner, } \\
\text { because Quick Cleaner.. }\end{array}$ & $\begin{array}{l}\text { Ladies, use Quick Cleaner } \\
\text { because Quick Cleaner is... }\end{array}$ \\
\hline
\end{tabular}

Tati, qui n'a par ailleurs jamais recours, dans le film, au gros plan pour cadrer les visages, fait usage d'un insert — forme de focalisation visuelle — , que les traducteurs ont décidé de rendre par un sous-titre - forme de focalisation linguistique par nature (du texte par opposition à une absence de texte). Ils passent donc outre la presque vacuité sémantique de la phrase afin de traduire au mieux cet effet voulu par Tati, dont la fonction est sans doute de signifier 
la place prépondérante que prend le marketing commercial dans la vie de ces gens à la fin des années 60 .

\section{Les traductions qui se veulent proches du texte-source}

Nous ne nous intéresserons pas ici aux traductions littérales qui rendent efficacement les intentions du dialogue d'origine, mais plutôt à celles qui, par excès de transcodage, finissent paradoxalement par aller à l'encontre du projet artistique du cinéaste. Nous avons montré qu'une bonne traduction du dialogue de Playtime ne pouvait faire l'économie, à notre sens, d'une réflexion sur la fonction du dialogue dans le film. On pressent notamment le risque qu'il y aurait à vouloir rendre les « sécrétions humaines » qui envahissent l'œuvre par des traductions littérales systématiques. On peut s'interroger, par exemple, sur la nécessité de traduire les dialogues de l'exemple 15. Dans la scène suivante, un organisateur de voyage s'adresse à ses clients :

Exemple 15. BFI $00: 09: 15 ;$ EG $00: 08: 43$

\begin{tabular}{|l|l|l|}
\hline DIALOGUE D'ORIGINE & TRADUCTION DE BFI & $\begin{array}{l}\text { TRADUCTION D'ECLAIR } \\
\text { GROUP }\end{array}$ \\
\hline $\begin{array}{l}\text { - Come on ladies, par ici. } \\
\text { Suivez-moi ! Alors you } \\
\text { stop here, please. Ladies, } \\
\text { please stop... Ah ! Alors } \\
\text { écoute : de là jusque là, le } \\
\text { Royal Hotel. }\end{array}$ & $\begin{array}{l}\text { From here to here: Royal } \\
\text { Hotel// }\end{array}$ & $\begin{array}{l}\text { From here to here : Royal } \\
\text { Hotel.// }\end{array}$ \\
\hline $\begin{array}{l}\text { Et alors de là jusque là, le } \\
\text { Moderne, hein ? } \\
\text {-Donc, bon voyage ! } \\
\text { Par ici ! }\end{array}$ & From here: the Moderne & $\begin{array}{l}\text { And here to here... the } \\
\text { Moderne.// }\end{array}$ \\
\hline
\end{tabular}

Quelle est la fonction de ces sous-titres ? S'agit-il de simples « jalons » destinés à préparer mentalement le public à sa découverte future du Royal Garden ? D'un effet de pointillisme, les sous-titreurs craignant de laisser défiler la bande-son trop longtemps sans proposer de traduction ? Au-delà du risque de l'alourdissement du sous-titrage, il nous semble que, dans le contexte précis de ce film, la volonté de tout traduire peut parfois même desservir son esthétique. Qu'en est-il, par exemple, avec l'exemple 16, dans lequel un serveur explique à son patron qu'il ne peut pas faire passer son poisson à travers le passe-plat car l'ouverture est trop étroite : 
Exemple 16. BFI $01: 05: 20$; EG $01: 04: 47$

\begin{tabular}{|c|c|c|}
\hline DIALOGUE D'ORIGINE & TRADUCTION DE BFI & $\begin{array}{l}\text { TRADUCTION D'ECLAIR } \\
\text { GROUP }\end{array}$ \\
\hline \multirow{6}{*}{$\begin{array}{l}\text { — Qu'est-ce qui va pas, } \\
\text { vous? } \\
\text { - Alors, regardez la } \\
\text { sauce. Je peux pas le } \\
\text { prendre par le haut (il } \\
\text { montre que la sauce } \\
\text { coule hors du plat). } \\
\text { Aaah! } \\
\text { — Passez dans l'autre } \\
\text { sens! } \\
\text { — Dans l'autre sens? } \\
\text { Alors là, vous allez voir } \\
\text { ! Dans l'autre sens, tenez } \\
\text { ! (La sauce coule de } \\
\text { nouveau hors du plat). } \\
\text { — (?). } \\
\text { — Alors passez le par la } \\
\text { porte! }\end{array}$} & \multirow[t]{6}{*}{ N.T. } & Look at the sauce. \\
\hline & & Turn it around. \\
\hline & & In the other direction? There. \\
\hline & & $\begin{array}{l}\text { The fish is } 50 . \\
\text { You have } 50 \text { here. }\end{array}$ \\
\hline & & It should go through. \\
\hline & & Then go through the door. \\
\hline
\end{tabular}

Dans le cas où un gag s'appuie sur des éléments visuels universels - dans la mesure, donc, où il peut être compris par n'importe quel individu (quelles que soient sa langue et sa culture) et où le langage utilisé à cette occasion par les personnages n'a pour fonction que de créer un effet de réel -, on peut légitimement se poser la question de savoir si sous-titrer le gag en question est la meilleure solution. Ici, bien que le dialogue français soit relativement long, la non-traduction ne pose a priori pas de problème de compréhension car la gestuelle des différents protagonistes indique aussi bien que leurs paroles ce qui est en train de se passer et ce qu'ils en pensent. De manière intéressante, EG fait un choix radicalement différent : il est assez clair ici que son rendu du dialogue est assez complet. Mais la question reste posée : la traduction ne crée-t-elle pas un effet de redondance par rapport à l'image ? Compte tenu de la nature essentiellement visuelle du gag, n'attire-t-on pas inutilement l'attention du spectateur sur un texte dont on pourrait facilement se passer ? Faut-il privilégier les éléments de nature sociolinguistique — qui ont évidemment leur importance mais qui peuvent être facilement compensés à un autre moment du film — ou l'élément comique visuel ? 


\section{Conclusion}

Létude montre assez clairement que l'hypothèse de la réduction drastique du dialogue se confirme chez les deux traducteurs, bien que BFI soit beaucoup plus radical que son concurrent sur ce point. Elle entérine aussi l'idée selon laquelle — sauf exception — le subtil jeu de focalisation / défocalisation opéré par Tati est parfois perdu à la traduction. Par ailleurs, elle témoigne du fait que dans les deux versions des traducteurs, l'appréhension synthétique de la bande-son (sous la forme de dialogues qui se chevauchent), fait place à des traductions qui relèvent davantage de l'appréhension analytique du sens. On gardera toutefois à l'esprit que dans le cas du sous-titrage, la bande sonore défile en même temps que les sous-titres, donnant en cela au spectateur un contexte permettant de relativiser la « dénaturation » engendrée par le texte écrit. Cela est particulièrement pertinent s'agissant d'une bande-son que l'on peut appréhender dans certaines scènes davantage comme un bruit que comme des répliques clairement articulées. Enfin, l'étude permet de mettre en évidence que dans certains contextes, la volonté de vouloir traduire un maximum de répliques peut aller à l'encontre du projet esthétique du cinéaste.

La comparaison des deux versions étudiées nous amène en outre à suggérer que les conceptions traductologiques des deux sous-titreurs au sujet de la fonction du dialogue diffèrent fortement, engendrant de ce fait deux constructions assez dissemblables de la « France de Tati ». Pour EG, le « bon » sous-titrage s'apparente à une traduction explicite et proche du texte de départ, d'où un plus grand respect dans son texte des caractéristiques sociolinguistiques et technolectales du dialogue de départ ; d'où, aussi, un rendu du détail plus fin que celui de son concurrent. Toutefois, faute peut-être d'une réflexion suffisante sur le rôle joué par la langue dans le film, cette orientation peut s'avérer contreproductive sur certains segments, en dénaturant les effets de focalisation ou de défocalisation recherchés par l'auteur, en faisant basculer le « bruit de la langue », la sécrétion linguistique et culturelle propre à cette France tatiesque, vers la banalité sémantique. Avec EG, le spectateur dispose donc de davantage de marqueurs pour reconstituer l'identité française vue par Tati ; en revanche, son souci de vouloir rendre autant de répliques que possible focalise parfois l'attention sur ce qui n'est qu'accessoire dans le regard du cinéaste. Moins proche de la lettre du texte, la version de BFI perd parfois en rigueur sémantique ce qu'elle gagne en liberté, tout en témoignant d'une réflexion intéressante sur la fonction esthétique du langage parlé dans le film. 


\section{Références}

CHIAro, Delia. (2009) "Issues in audiovisual translation." Dans: Munday, Jeremy (éd.) 2009. The Routledge Companion to Translation Studies. Londres \& New York: Routledge, édition révisée, pp. 141-164.

Chion, Michel. (2009) Jacques Tati. Paris: Cahiers du cinéma.

CORnu, Jean-François. (2011) "Le public? Quel public? De l'influence négligeable des spectateurs sur les stratégies de traduction audiovisuelle des films en France." Dans: Şerban, Adriana \& Jean-Marc Lavaur (éds.) 2011. Traduction et médias audiovisuels. Villeneuve d'Ascq: Presses Universitaires du Septentrion, pp. 21-35.

De Linde, Zoé \& Neil KaY. (1999) The Semiotics of Subtitling. Manchester: St. Jerome Publishing.

DíAZ-CinTAS, Jorge. (2005) "Back to the Future in Subtitling." Dans: MuTra: Challenges of Multidimensional Translation. Saarbrücken: EU High Level Scientific Conference Series. Version électronique: https://www.euroconferences.info/proceedings/2005_Proceedings/2005_proceedings.html

Dondey, Marc. (2002) Tati. Paris: Ramsay.

EdE, François \& Stéphane GoudeT. (2002) Playtime. Paris: Cahiers du cinéma.

ETIEMBle, René. (1994) Parlez-vous franglais? Édition révisée. Paris: Gallimard.

FIESCHI, Jean-André. (1996) La voix de Jacques Tati. Mulhouse: LimeLight.

GeOrgakopoulou, Panayota. (2009) "Subtitling for the DVD Industry." Dans:

Díaz-Cintas, Jorge \& Gunilla Anderman (éds.) 2009. Audiovisual Translation:

Language Transfer on Screen. New York: Palgrave Macmillan.

Gottlieb, Henrik. (1994) "Subtitling: Diagonal Translation." Dans: Perspectives,

Studies in Translatology 2:1, pp. 101-21.

GOUdET, Stéphane. (2002) Jacques Tati: de François le facteur à Monsieur Hulot.

Paris: Cahiers du cinéma.

Guerand, Jean-Philippe. (2007) Jacques Tati. Paris: Gallimard.

LAUFER, Laura. (2002) Jacques Tati. Paris: Les Éditions de l'If.

LAVAUR, Jean-Marc, \& Adriana ŞERBAN (éds.) (2008) La traduction audiovisuelle.

Bruxelles: De Boeck.

PÉREZ-GonZÁLEZ, Luis. (1998) "Audiovisual translation." Dans: Baker, Mona \&

Gabriela Saldanha (éds.) 1998. Routledge Encyclopedia of Translation Studies.

Londres: Routledge.

PRÉDAL, René. (2007) Esthétique de la mise en scène. Condé-sur-Noireau: Cerf-Corlet.

SELF, Robert T. (2001) Robert Altman's Subliminal Reality. Minneapolis: University of Minnesota Press.

THORET, Jean-Baptiste. (2006) Cinéma contemporain. Mode d'emploi. Paris: Flammarion. 
TOURY, Gideon. (1983) "Sharing Relevant Features: An Exercise in Optimal Translating." Meta: journal des traducteurs 28:2, pp. 116-129.

\section{Filmographie}

Jour de fête (Jacques Tati 1949)

Les grandes heures: "Un comique de vocation: Jacques Tati" (Ina/Radio France 2009)

Les Quatre cents coups (François Truffaut 1959)

Les Vacances de Monsieur Hulot (Jacques Tati 1953)

Parade (Jacques Tati 1974)

Playtime (Jacques Tati 1967)

Playtime (Jacques Tati 1967 / BFI 2010).

Playtime (Jacques Tati 1967 / Studiocanal 2014)

\section{NOTE BIOGRAPHIQUE / BIONOTE}

SÉBASTIEN GOUTTEFANGE enseigne actuellement l'anglais en lycée et effectue une thèse dans le domaine de la traduction à l'Université Paul-Valéry Montpellier 3. Titulaire de l'agrégation, il est membre de l'équipe de recherche EMMA. Sa recherche porte sur le sous-titrage des films de Robert Altman. Il s'intéresse tout particulièrement au lien entre l'esthétique du film et la traduction audiovisuelle.

SÉBASTIEN GOUTTEFANGE teaches English in a high school in France and is a doctoral student in translation at Paul Valéry University, Montpellier. He is a member of the research team EMMA and is a holder of the agrégation in English. His research focuses on the subtitling of Robert Altman's movies. He is particularly interested in the link between film aesthetics and audiovisual translation. 